\title{
Myoclonus of ipsilateral upper extremity after ultrasound-guided supraclavicular brachial plexus block with mepivacaine
} Korean Journal of Anesthesiology

\author{
Bong Jin Kang ${ }^{1}$, Jaegyok Song ${ }^{1}$, Sung-Mi Ji ${ }^{1}$, and Jong Pil Kim ${ }^{2}$ \\ Departments of ${ }^{1}$ Anesthesiology and Pain Medicine, ${ }^{2}$ Orthopedic Surgery, Dankook University College of Medicine, \\ Cheonan, Korea
}

Brachial plexus blocks are generally used for various surgical and analgesic procedures. Myoclonus after regional anesthesia has rarely been reported, but there have been a few reports of myoclonus after a peripheral nerve block. We report a very rare case of myoclonus after a supraclavicular brachial plexus block in a healthy patient with a brief review of literature.

A 22-year-old woman (height: $160 \mathrm{~cm}$, body weight: $46 \mathrm{~kg}$, American Society of Anesthesiologists physical status I) was scheduled for a capsular release operation. There was nothing specific in her past medical history. She had previously undergone an uncomplicated left hand procedure under general anesthesia with no anesthesia-related adverse events. Preoperative evaluations were also normal. A supraclavicular brachial plexus block was planned for the anesthesia. Electrocardiography, noninvasive blood pressure, and pulse oximetry were monitored.

Before induction of anesthesia, midazolam $2 \mathrm{mg}$ and fentanyl $50 \mu \mathrm{g}$ were intravenously injected for sedation and pain relief. At the beginning of nerve block, her blood pressure and heart rate were $120 / 75 \mathrm{mmHg}$ and 65 beats/min.

Corresponding author: Jaegyok Song, M.D., Ph.D.

Department of Anesthesiology and Pain Medicine, Dankook University College of Medicine, 201, Manghyang-ro, Dongnam-gu, Cheonan 31116, Korea

Tel: 82-41-550-6819, Fax: 82-41-551-9330

Email: drjack@nate.com

ORCID: https://orcid.org/0000-0002-4727-6296

This article includes supplemental video clips.

Received: January 10, 2017.

Revised: January 20, 2017 (1st); January 26, 2017 (2nd).

Accepted: January 27, 2017.

Korean J Anesthesiol 2017 October 70(5): 577-579

https://doi.org/10.4097/kjae.2017.70.5.577
A supraclavicular brachial plexus block was performed under ultrasonographic guidance with a $5 \mathrm{~cm}$ standard bevel needle (Profi needle ${ }^{\mathrm{TM}}$, Shinchang medical Co., Seoul, Korea). We injected $1.5 \%$ mepivacaine $40 \mathrm{ml}$ after confirming no aspiration of blood. She did not complain of any severe paresthesia or injection pain during the procedure. Fifteen minutes after procedure, we confirmed successful sensory and motor block of brachial plexus and $5 \mathrm{~L} / \mathrm{min}$ of oxygen was supplied via facial mask during the operation. An additional $1 \mathrm{mg}$ of midazolam was given intravenously for sedation. The patient was stable during the surgery and the duration of surgical procedure was about 40 minutes. After the surgical procedure, the patient was transferred to a recovery room. One hour after the injection of the local anesthetic, she showed a shivering motion without any chilling sensation. The body temperature was $36.7^{\circ} \mathrm{C}$. Meperidine 25 mg was intravenously injected to relieve shivering and $5 \mathrm{~L} / \mathrm{min}$ of oxygen was supplied via facial mask. However, her shivering continued for about 15 minutes and the patient began to show mild agitation. Thirty minutes after meperidine injection, she showed involuntary movement of left arm, while the other body parts were under control (Video 1). The patient was unable to suppress the movement intentionally but remained conscious and communicated appropriately. She did not complain of pain or any other discomfort and there were no other neurologic symptoms. The driving force for the abnormal movement was from the surrounding muscles of the shoulder with some contribution from the muscles of the elbow joint. We consulted to neurology and the patient was treated with an intravenous injection of midazolam $5 \mathrm{mg}$ but it was not effective. The movement continued while patient was sedated with midazolam. The neurologist examined the patient and about 20 minutes after midazolam injection, lorazepam $4 \mathrm{mg}$ was administered intravenously but it was also not effective. The abnormal movement did

(c) This is an open-access article distributed under the terms of the Creative Commons Attribution Non-Commercial License (http://creativecommons.org/ licenses/by-nc/4.0/), which permits unrestricted non-commercial use, distribution, and reproduction in any medium, provided the original work is properly cited. 


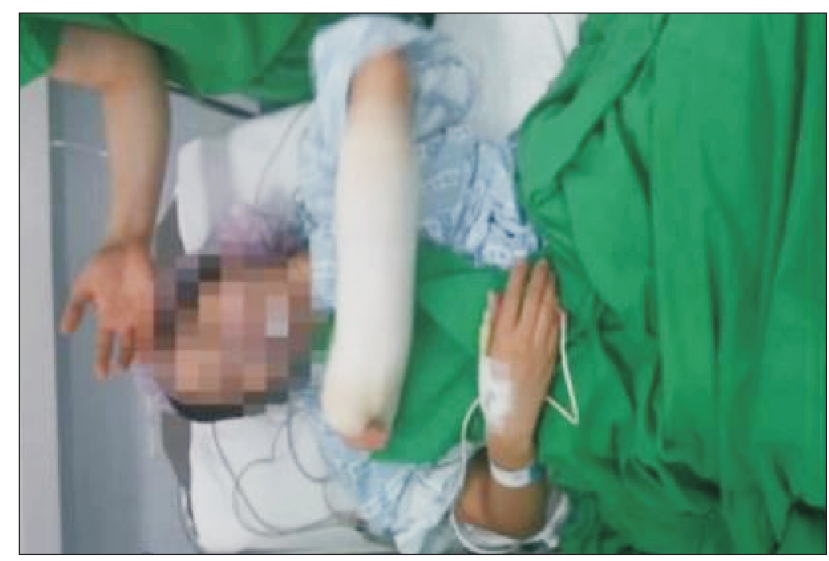

Video 1 . The patient showed involuntary movement of left arm, while the other body parts were intact. The patient was remained conscious and communicated appropriately and also could follow the neurologist's orders. The whole left arm moved back and forth around the shoulder joint quickly and irregularly also with some repetitive flexion-extensions of the elbow. The patient was unable to suppress the movement intentionally and did not complain of pain or any other discomfort and there was no other neurologic symptom.

not have any aggravating or relieving factors either. Laboratory findings were unremarkable. Vital signs were stable during the midazolam and lorazepam treatment and the patient was closely monitored to prevent drug induced complications including respiratory depression.

The movement decreased gradually and completely disappeared 10 hours after the operation without further treatment. Brain magnetic resonance imaging showed no significant abnormal lesion in brain parenchyma.

On next day, the patient experienced the same type of involuntary movement 3 times lasting for about 5 minutes each. The symptoms disappeared spontaneously without any medication. No other neurologic symptom was reported. The patient was discharged on the 5th postoperative day without complication. Telephone interview was performed 1 month after discharge, and there had not been any more episodes of neurologic symptoms.

Myoclonus is a medical sign that a brief, involuntary twitching of a muscle or a group of muscles. The classification of myoclonus is scheme based on the neuroanatomical origin of electrical discharge including cortical (most common type), subcortical, spinal or peripheral myoclonus [1].

Our case displays several unique clinical characteristics. When the movement disorder had started, the patient's mental state was alert and cooperative. The myoclonus was confined to the single arm that was anesthetized with local anesthetic injection. We used midazolam and lorazepam to treat the myoclonus but it was not effective. We used midazolam, fentanyl, and meperidine during the anesthesia which had been known to induce myoclonus. However, the characteristics of this drug induced myoclonus were generalized or bilateral involvement of the limbs [2]. The dose of mepivacaine was relatively high for the patient's body mass, whereas the patient did not show any symptom and sign of local anesthetic toxicity. Thus, we could rule out seizure disorders, cortical and subcortical myoclonus, and drug induced myoclonus $[2,3]$. Our patient presented clinical symptoms suggestive of spinal myoclonus; the rhythmic, nonstimulus nature and frequency $(2-3 \mathrm{~Hz})$ of her unilateral upper limb movements. Unfortunately, the symptom had ceased before we could perform electromyography. However, the patient did not show any symptom and sign of cervical neuroaxial anesthesia. Although the mepivacaine dose was high, the possibility of drug migration into subarachnoid space via cervical nerve roots was very low [4].

Another theory is that peripheral nerve trauma or nerve entrapment may have induced peripheral myoclonus. Assal et al. [5] reported involuntary movement after the injury to the cutaneous branch of the deep peroneal nerve that was rhythmic and was not painful which was similar to our case. We performed the brachial plexus block under ultrasonographic guidance and the patient did not complain severe paresthesia or pain during the block. However, we cannot exclude the possibility of nerve injury that may cause the transient myoclonus. We used 26 gauge $(G)$ standard bevel needle instead of short bevel needle. The anesthesiologist who performed the nerve block was not well experienced and felt some difficulty in noticing the tip of needle with ultrasonography because the needle is thinner than the commonly used $22 \mathrm{G}$ short bevel needle that resulted in an overdose of mepivacaine, too. Also, this may have increased the risk of nerve damage. Therefore, the position of the needle tip and the path of needle insertion must be carefully monitored when a nerve block is performed under ultrasonographic guidance to prevent nerve injury.

\section{References}

1. Caviness JN, Brown P. Myoclonus: current concepts and recent advances. Lancet Neurol 2004; 3: 598-607.

2. Lai HC, Hu MH, Liaw WJ, Lu CH, Huang GS. Atypical involuntary movements following fentanyl anesthesia. J Clin Anesth 2013; 25 : 58-61.

3. Ozcan B, Kavurt S, Yucel H, Bas AY, Demirel N. Rhythmic myoclonic jerking induced by midazolam in a preterm infant. Pediatr Neurol 2015; 52: e9. 
4. Lev A, Korn-Lubezki I, Steiner-Birmanns B, Samueloff A, Gozal Y, Ioscovich A. Prolonged propriospinal myoclonus following spinal anesthesia for cesarean section: case report and literature review. Arch Gynecol Obstet 2012; 286: 271-2.

5. Assal F, Magistris MR, Vingerhoets FJ. Post-traumatic stimulus suppressible myoclonus of peripheral origin. J Neurol Neurosurg Psychiatry 1998; 64: 673-5. 